\title{
Optimal Stacking Sequence Design of Laminated Composite Shells
}

\author{
C.W. Kim and W. Hwang \\ Department of Mechanical Engineering \\ Pohang University of Science and Technology \\ Pohang 790-784, KOREA
}

\begin{abstract}
An optimization procedure is proposed for the design of cylindrical composite shell having midplane symmetry and subjected to axial force and internal pressure. Tsai-Wu failure criterion is taken as objective function while the stacking sequence represents the design variable. The optimal design formulation based on the state space method is adopted and solution procedure is described with the emphasis on the method of calculating the design sensitivities. A gradient projection algorithm is employed for the optimization process. Numerical results are presented for the several test problems.
\end{abstract}

KEYWORDS: Optimization; Cylindrical composite shell; Design sensitivity; Min-max problem; Failure-criterion; State space method.

\section{INTRODUCTION}

Composite materials in mechanical, aerospace, and other branch of engineering are increasingly used due to their excellent weight saving and the ease of tailoring. In spite of tremendous progress in analytical capability to analyze the behavior of composite materials and structures, there is a lack of design models which may allow efficient tailoring of their properties to specific requirements for structural components. To improve this long-pending problem, the optimum design of composite materials has been a subject of research for many years[1-6]. Several papers have been published on the optimum design of composite plates[1,2,3] but not many on the composite shells $[4,5,6]$. The usual object of optimum design is to design layer thickness or layer orientation which will give the minimum weight[1] or the maximum stiffness[2,3]. In this paper, strength of cylindrical composite shell structures is considered and the layer angles are taken as the design variables. State space method[8] is used to formulate optimal design problem. To treat worst-case design, optimal design problem is formulated as Min-max problem considering environmental parameters. The design sensitivities of functions[9] with respect to fiber orientations are calculated using adjoint variable method and state equations. Its results are verified with finite difference method(F.D.M.). Based on design sensitivity information, Gradient projection(GRP) algorithm is combined with the optimality criterion which may minimize the failure index of failure criterion[10]. Several examples are given to demonstrate the applications of proposed method. The optimal design results are compared with netting analysis which gives 
ideal angle $54.735^{\circ}$ under internal pressure.

\section{ANALYSIS}

The configuration of cylindrical composite shell and cross section are presented in Figure 1. The cylindrical coordinate system is defined in Figure 2. Based on Kirchhoff-Love hypothesis, the cylindrical wall is assumed to be thin and to have linear elastic behavior. It is assumed further that the lamina of the cylinder is stacked alternatively with $+\theta$ and $-\theta$ angles. For this thin shell, the displacement field is assumed to be given by:

$$
\begin{aligned}
& u(x, \theta, z)=u^{o}(x, \theta)+z F_{x}^{o}(x, \theta) \\
& v(x, \theta, z)=v^{o}(x, \theta)+z F_{\theta}^{o}(x, \theta) \\
& w(x, \theta, z)=w^{o}(x, \theta)
\end{aligned}
$$

Hooke's law in conjunction with the strain-displacement relation and the stress and moment resultant definition yields the following constitutive relations for the cylindrical composite shells[7]:

$$
\left[\begin{array}{l}
N_{x} \\
N_{\theta} \\
N_{x \theta} \\
M_{x} \\
M_{\theta} \\
M_{x \theta}
\end{array}\right]=\left[\begin{array}{llllll}
A_{11} & A_{12} & A_{16} & B_{11} & B_{12} & B_{16} \\
A_{12} & A_{22} & A_{26} & B_{12} & B_{22} & B_{26} \\
A_{16} & A_{26} & A_{66} & B_{16} & B_{26} & B_{66} \\
B_{11} & B_{12} & B_{16} & D_{11} & D_{12} & D_{16} \\
B_{12} & B_{22} & B_{26} & D_{12} & D_{22} & D_{26} \\
B_{16} & B_{26} & B_{66} & D_{16} & D_{26} & D_{66}
\end{array}\right]\left[\begin{array}{c}
\varepsilon_{x}^{o} \\
\varepsilon_{\theta}^{o} \\
\gamma_{x \theta}^{3} \\
\kappa_{x} \\
\kappa_{\theta} \\
\kappa_{x \theta}
\end{array}\right]
$$

The partially inverted form of the above constitutive relations can be found as follows[11]:

$$
\left[\begin{array}{c}
\varepsilon^{\rho} \\
M
\end{array}\right]=\left[\begin{array}{cc}
A^{*} & B^{*} \\
-B^{*} & D^{*}
\end{array} \mid\left[\begin{array}{c}
N \\
\kappa
\end{array}\right]\right.
$$

where $A^{*}=A^{-1}, B^{*}=-A^{-l} B$, and $D^{*}=D-B A^{-l} B$. The equilibrium and compatibility equations are satisfied when the $\theta$-direction resultant force, $N_{\theta}$ is [11]:

$$
N_{\theta}=R p
$$

Then, the total torque $\mathrm{T}$ applied to the shell can be obtained from the above equation and the equilibrium of moments 


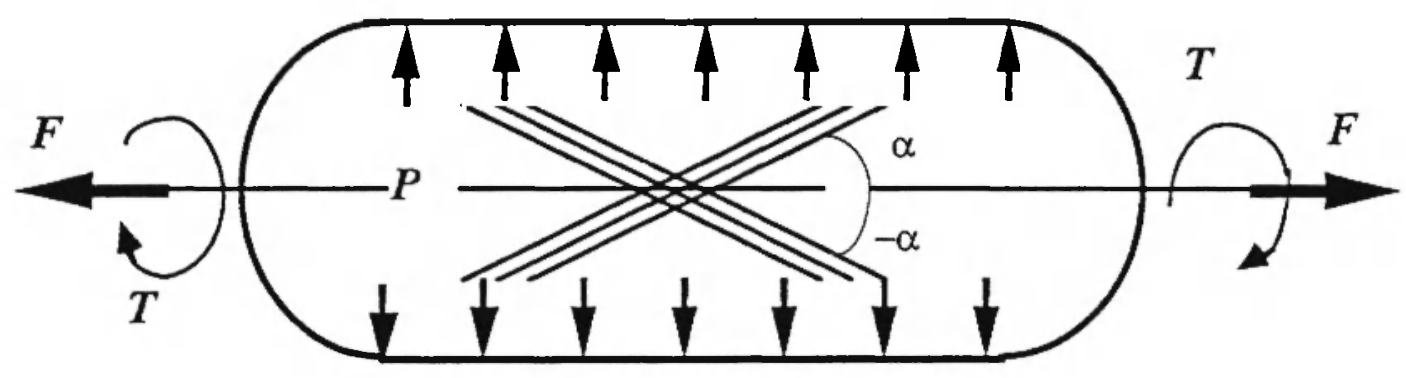

(a)

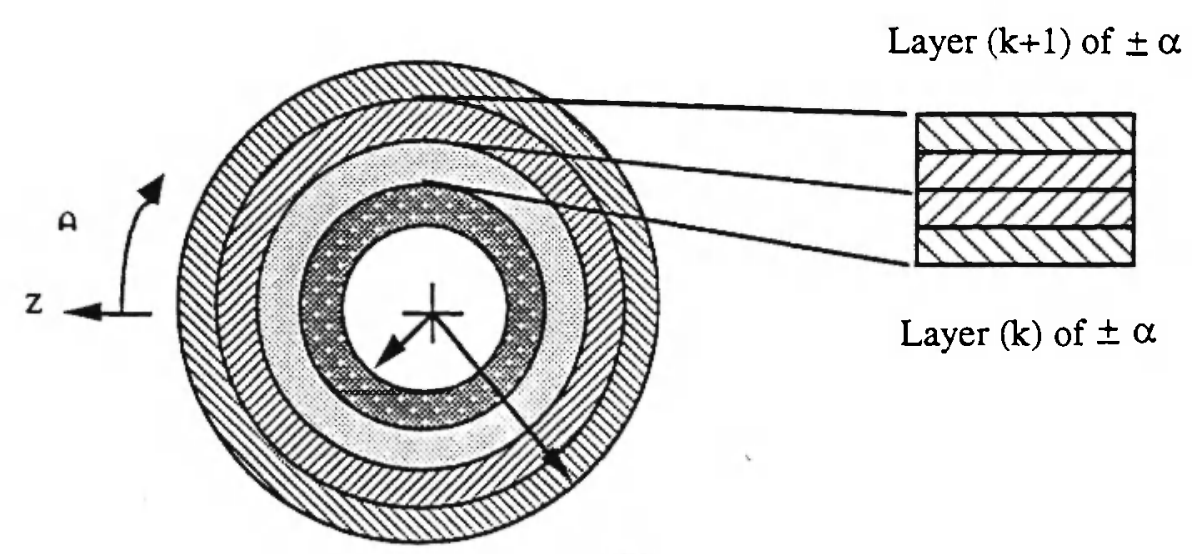

(b)

Fig.1 Configuration of cylindrical composite shell and cross section (a) configuration, (b) cross section

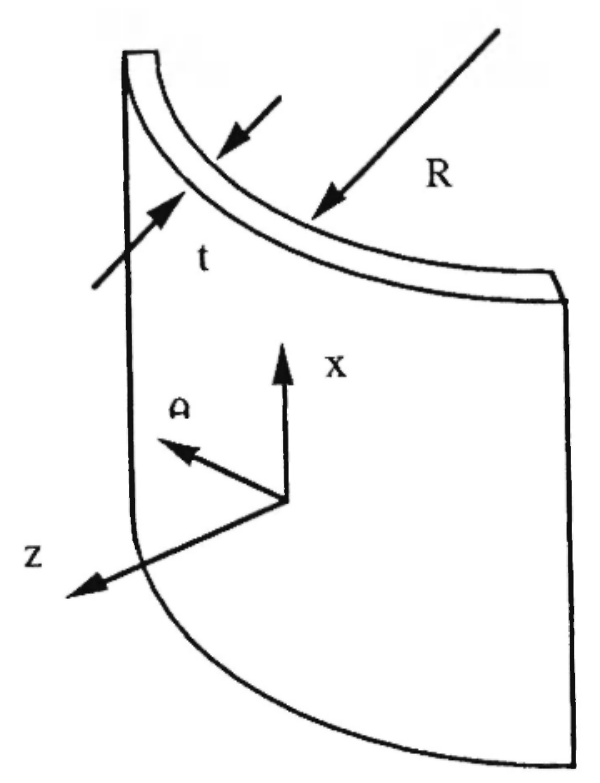

Fig.2 Cylindrical coordinate 


$$
\begin{aligned}
T & =2 \pi R\left(R N_{x \theta}+M_{x \theta}\right) \\
& =2 \pi R\left(R N_{x \theta}-B_{16}^{*} N_{x}-B_{26}^{*} R p\right)
\end{aligned}
$$

Substituting Equations (4) and (5) into Equation (3) yields following strain relations:

$$
\begin{array}{r}
\varepsilon_{x}^{n}=\left(A_{11}^{*}+A_{16}^{*} K_{1}\right) N_{x}+\left(A_{12}^{*}+A_{16}^{*} K_{2}\right) R p+A_{16}^{*} K_{3} T \\
\varepsilon_{\theta}^{n}=\left(A_{12}^{*}+A_{26}^{*} K_{1}\right) N_{x}+\left(A_{22}^{*}+A_{26}^{*} K_{2}\right) R p+A_{26}^{*} K_{3} T \\
\gamma_{x \theta}^{o}=\left(A_{16}^{*}+A_{66}^{*} K_{1}\right) N_{x}+\left(A_{26}^{*}+A_{66}^{*} K_{2}\right) R p+A_{66}^{*} K_{3} T
\end{array}
$$

For symmetric layups $\left(\boldsymbol{B}_{i j}=0\right)$, the stress-strain relations in the $\mathrm{k}$-th layer are:

$$
\left\{\begin{array}{c}
\sigma_{x} \\
\sigma_{\theta} \\
\sigma_{x \theta}
\end{array}\right\}^{(k)}=\left[\begin{array}{lll}
\bar{Q}_{11} & \bar{Q}_{12} & \bar{Q}_{16} \\
\bar{Q}_{12} & \bar{Q}_{22} & \bar{Q}_{26} \\
\bar{Q}_{16} & \bar{Q}_{26} & \bar{Q}_{6 \sigma}
\end{array}\right]^{(k)}\left\{\begin{array}{c}
\hat{c}_{x} \\
\vec{c}_{\theta} \\
\gamma_{x \theta}
\end{array}\right\}
$$

\section{PROBLEM FORMULATION}

\subsection{Optimization Procedure}

The optimization problem that we consider here is to maximize the strength of cylindrical composite shells for given layer thickness. Each layer angle is taken as a design variable. To consider strength, Tsai-Wu quadratic failure criterion is used. State space method is used to formulate optimal design problem. To treat worst-case design, optimal design problem is formulated as Min-max problem considering environmental parameter, $\alpha$ In this problem, maximum failure index through thickness is treated as environmental parameter. The optimization problem can be expressed in mathematical form as follows:

$$
\text { Minimize } \underset{\alpha_{\min }<\alpha<\alpha_{\max }}{\text { Maximium }} F\left(\sigma_{1}, \sigma_{2}, \tau_{12}\right)
$$

with the state equation $h(z, b, \alpha)=0$

$$
F=\left(\frac{1}{X}-\frac{1}{X^{\prime}}\right) \sigma_{1}+\left(\frac{1}{Y}-\frac{1}{Y^{\prime}}\right) \sigma_{2}+\frac{1}{X X^{\prime}} \sigma_{1}^{2}+\frac{1}{Y Y^{\prime}} \sigma_{2}^{2}+\frac{1}{S^{2}} \tau_{12}^{2}-\sqrt{F_{11} F_{22}}
$$

where, $F\left(\sigma_{l}, \sigma_{2}, \tau_{12}\right)$ is Tsai-Wu failure criterion and defined as failure index(F.I.). In some 
cases, the above Tsai-Wu failure criterion is not applicable especially, when the unidirectional angle ply laminates are subjected to axial force or torsion. For instance, minus failure index values occur near $20^{\circ}$ to $30^{\circ}$ when the unidirectional angle ply laminates are subjected to tensile loading $N_{x}=0.5 \mathrm{MN} / \mathrm{mm}$. As a result, the optimum angle, which gives minimum failure index, is found to be $20.080^{\circ}$. This result is not only far from reality but also meaningless in the view point of physical meaning. To avoid this inconsistency, it is assumed that transverse compressive strength is same as tensile strength i.e., $Y^{\prime}=Y$.

Min-max design formulation can be reduced to a parametric optimal design formulation using a dummy design variable $b(j+1)$ and a new constraints. The reduced parametric formulation can be written as follows:

$$
\begin{aligned}
& \text { Minimize } \Psi_{o}=b(j+1) \\
& \text { subject to } \Psi_{i}=\underset{\alpha_{\min }<\alpha<\alpha_{\max }}{\operatorname{Maxmum}} F\left(\sigma_{1}, \sigma_{2}, \tau_{12}\right)-b(j+1)<0 \\
& \text { with the state equations } \quad h(z, b, \alpha)=0
\end{aligned}
$$

\subsection{Sensitivity Analysis}

Every iterative algorithm for solving optimization problem requires design sensitivities. In this paper, based on adjoint variable method, the analysis procedures of cylindrical composite shells are treated as a set of state equations which may be used to calculate design sensitivities. The state equations can be formulated by Equations (3), (6), and (7). The resulting state equations are,

$$
\begin{aligned}
& h_{1}^{T}=\{\bar{Q}\}-f(\theta)=0 \\
& h_{2}^{T}=\{\boldsymbol{A}\}-f(\bar{Q})=0 \\
& h_{3}^{T}=\left\{\boldsymbol{A}^{-1}\right\}-f(\boldsymbol{A})=0 \\
& h_{4}^{T}=\left\{\varepsilon^{\rho}\right\}-f\left(\boldsymbol{A}^{-1}\right)=0 \\
& h_{5}^{T}=\{\sigma\}-f\left(\bar{Q}, \varepsilon^{\rho}\right)=0
\end{aligned}
$$

In the above equations, the transformed stiffness matrix, extensional stiffness matrix, transformed stiffness matrix, strain, and stress are state variables $(z)$, while $\theta$ represents design variables $(b)$. In adjoint variable method, the design sensitivity vector $l^{i}$ of a function $\Psi_{i}(z, b)$ can be written[8]: 


$$
\stackrel{i}{i}=\frac{\partial \Psi_{i}^{T}}{\partial b}-\frac{\partial h^{T}}{\partial b} \lambda^{i}
$$

The adjoint variable vector $\lambda^{\prime}$ is calculated from the solution of following adjoint equation

$$
J^{T} \lambda^{i}=\frac{\partial \Psi_{i}^{T}}{\partial z}
$$

where $J$ denotes the Jacobian matrix and $J=\frac{\partial h}{\partial z}\left[z^{o}, b^{o}\right]$. The design sensitivities can be calculated by the above Equations (12) and (13).

\section{NUMERICAL RESULTS AND DISCUSSION}

Some examples are presented to demonstrate the effect of optimization of stacking sequence on the strength of composite cylindrical shell. The optimization problem stated in Equation (10) will be solved by GRP algorithm with the design sensitivity information. A conceptual flow chart for solving the process of optimal design problem is given in Figure 3. In calculating numerical solution, The inner radius, $R_{t}$ of cylindrical composite shell is $100 \mathrm{~mm}$ and following

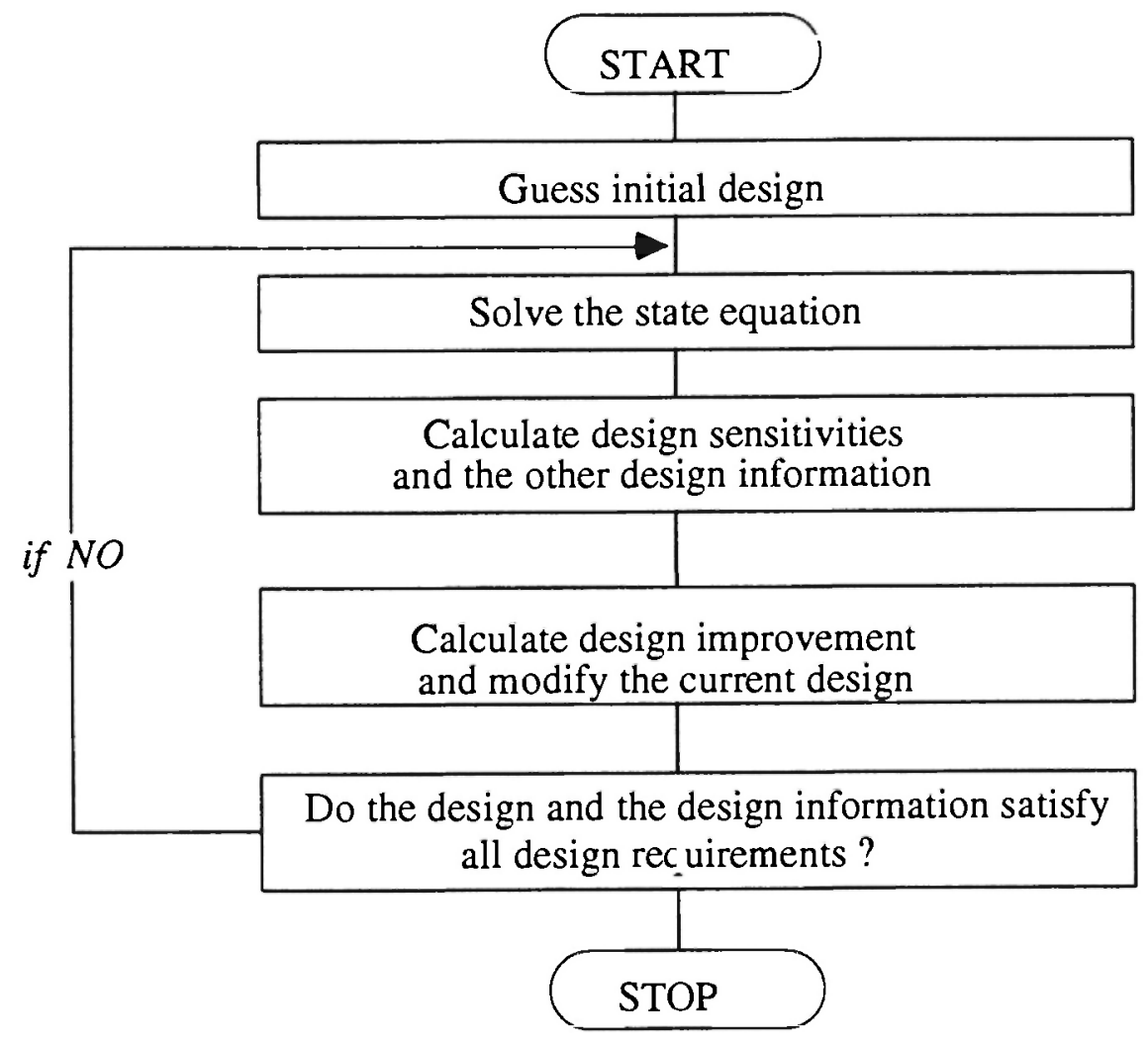

Fig. 3 Flow chart of optimization process 
material properties of T300/5208 graphite/epoxy are applied:

$$
\begin{array}{lll}
\mathrm{E}_{11}=181 \mathrm{GPa} \mathrm{E}_{22}=10.30 \mathrm{GPa} & \mathrm{G}_{12}=7.17 \mathrm{GPa} & v_{12}=0.28 \\
\mathrm{X}=1500 \mathrm{MPaX}=1500 \mathrm{MPa} & \mathrm{Y}=40 \mathrm{MPa} & \mathrm{Y}^{\prime}=246 \mathrm{MPa} S=68 \mathrm{MPa}
\end{array}
$$

\subsection{Sensitivity Analysis}

The design sensitivities for $[-45 / 45]_{2 S}$ laminated cylindrical shell subjected to internal pressure $p=10 \mathrm{MPa}$ are calculated and the results are tabulated in Table 1. The sensitivities evaluated by F.D.M. with various step size are also compared with present design sensitivity in Figure 4. The comparison results show that present design sensitivity calculated by adjoint variable method is very accurate. It can be seen that all the results evaluated by F.D.M. give reliable design sensitivities for a relatively wide range of step size from $10^{-7}$ to $10^{-3}$. However, in certain ranges of step size the large amounts of numerical error are observed when the design sensitivities are evaluated by F.D.M.

Table 1 Design Sensitivity of $[+45]_{2 s}$ Laminated Shell ( step size is $0.001^{\circ}$, internal pressure $p$ is $10 \mathrm{MPa}$ )

\begin{tabular}{cccccc}
\hline$i$ & $b_{i}$ & $l_{i}^{1}$ & $T_{i}^{()}$ & $T_{i}^{(b)}$ & $T_{i}^{(c)}$ \\
\hline 1 & 45 & -70.2377 & -70.2375 & -70.2380 & -70.2377 \\
2 & -45 & 55.5797 & 55.5794 & 55.5800 & 55.5797 \\
3 & 45 & -44.8316 & -44.8324 & -44.8307 & -44.8316 \\
4 & -45 & 55.5797 & 55.5794 & 55.5800 & 55.5797 \\
5 & & -1.0000 & -1.0000 & -1.0000 & -1.0000 \\
\hline
\end{tabular}

$l_{i}^{l}:$ adjoint variable method

$\tau_{i}^{(b)}$ : backward finite difference scheme $l_{:}^{(f)}:$ forward finite difference scheme $I_{i}^{(c)}$ : central finite difference scheme 


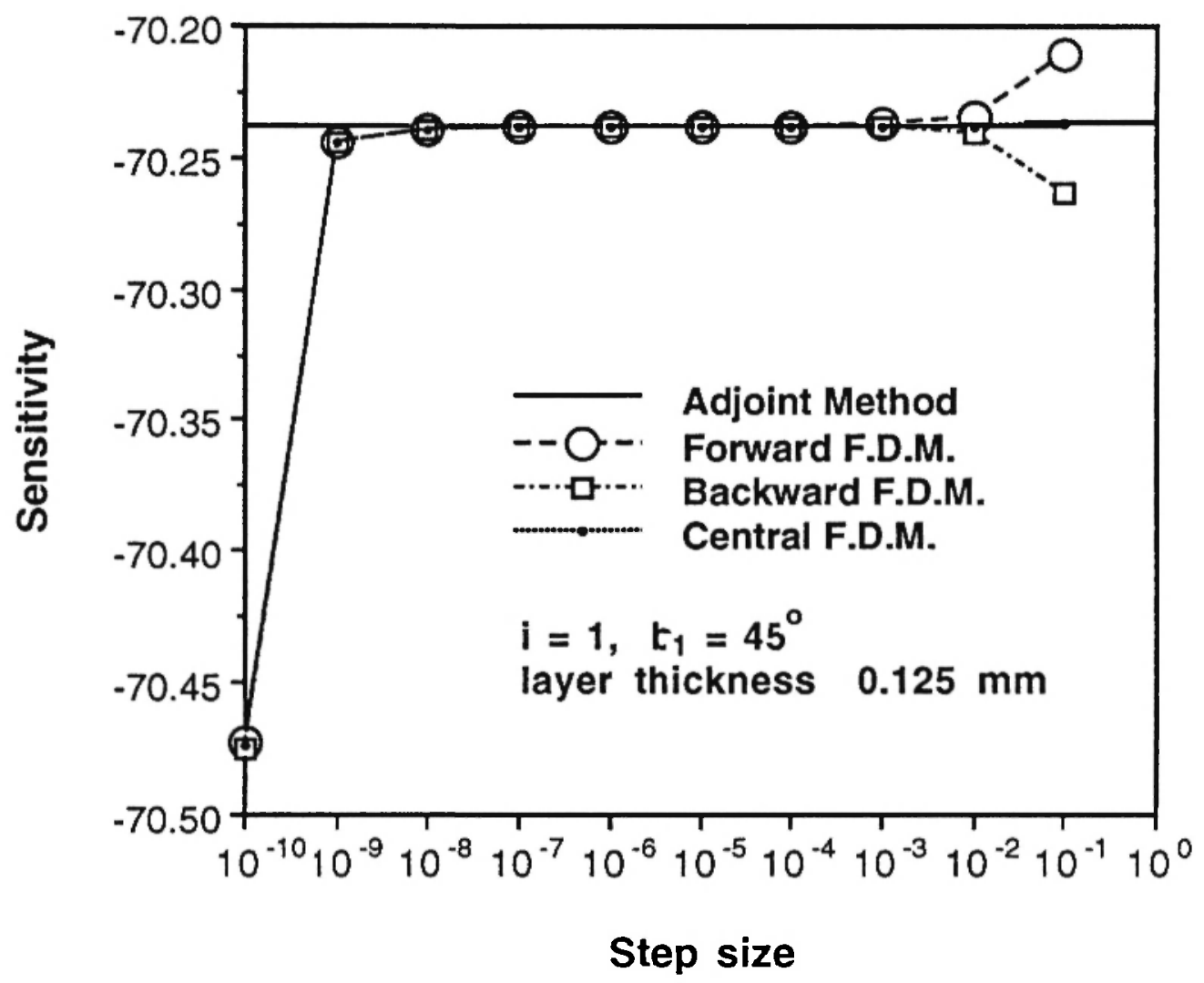

Fig. 4 Variation of design sensitivity of $[ \pm 45]_{2 S}$ laminated shell with internal pressure, $10 \mathrm{MPa}$

\subsection{Illustrative Examples}

The results have been given for the cases when cylindrically laminated shell structures are subjected to internal pressure and a simultaneous action of internal pressure and axial force.

To verify present method, optimization of $\left[ \pm \theta_{1} l_{ \pm} \theta_{2}\right]_{S}$ laminated shell subjected to tensile loading $\left(N_{x}=10 \mathrm{MN} / \mathrm{mm}\right)$ is investigated. It is obvious that the maximum strength of the laminated shell occurs when the all layer angles are $0^{\circ}$ under tensile loading. The same result is obtained in this study. Figure 5.(a) shows three dimensional plot and contours of the objective function with respect to design variables. It can be seen in the figure that minimum failure index, which implies the maximum strength, is found in $[0]_{2 s}$ laminated shell. It is also found that the laminated shell with all transverse fiber orientation $\left(90^{\circ}\right)$ has maximum failure index as expected. Present method is examined again by the optimization of $[ \pm \theta]_{s}$ and $\left[ \pm \theta_{1} / \pm \theta_{2}\right]_{s}$ laminated shell under torsional loading $(T=10 \mathrm{MN}-\mathrm{mm})$. The optimized stacking sequence results are $[+44.691]_{S}$ 

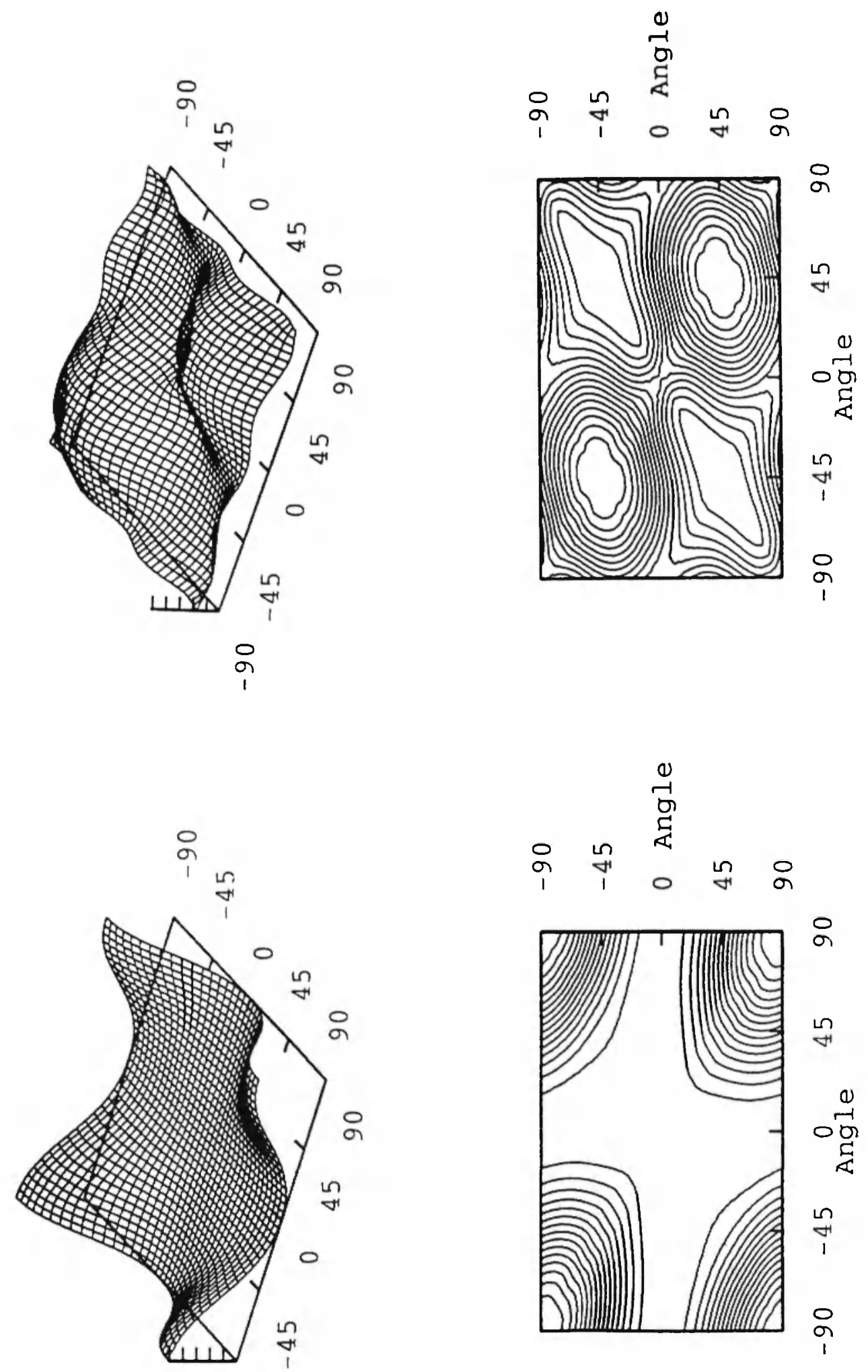

운 
and $[ \pm 44.691 / \pm 42.972]_{S}$ for the laminated shells $[ \pm \theta]_{S}$ and $\left[ \pm \theta_{1} / \pm \theta_{2}\right]_{S}$, respectively. These results almost coincide with analytical solution which gives $[ \pm 45]_{2 S}$ for maximum strength. It can be also seen in the plots of objective function presented in Figure 5.(b). The optimal design of the cylinders with various laminate thickness is sought. The result showed that the optimal angle do not change with increasing $R_{o} / R_{i}$ ratio.

The optimization results of cylindrical shell under internal pressure $(p=10 \mathrm{Mpa})$ are presented in Table 2. The multilayer optimum winding angle combinations with various $R_{o} / R_{i}$ ratios and maximum failure index of each case are obtained. The maximum failure indices of the optimized multilayer cylinders are compared with optimized single layer case. The comparison results are shown in Figure 6. It can be seen that the failure indices decrease as the number of layers increases. Present optimization analysis is compared with netting analysis which predicts $54.735^{\circ}$ as optimal winding angle for maximum strength. The comparison results are presented in Figure 7 and 8 for the thickness ratio 1.005 and 1.10, respectively. It is found that present analysis provides lower failure index than netting analysis. Table 3 represents the optimization results of the cylinder subjected to both internal pressure and axial force simultaneously. The hoop-to-axial force ratios are pre-selected. The resultant force ratio chosen are (1:0), (4:1), (2:1), (1:1), (1:2), $(1: 4)$, and $(0: 1)$. It is found that the optimal angles are $[90]_{2 s}$ in both laminated shells when the hoop-to-axial resultant force ratio is (1:0). It can be readily noticed that these results are the same as analytical solution. Another optimization example is given in Table 4. In this case, the two inner layer angles are fixed while the two outer angle, $+\theta$ treated as single design variable.

\section{CONCLUDING REMARKS}

An efficient approach for strength optimization of cylindrical composite shells subjected to internal pressure and axial forces is presented. The layer angles could be regarded as design variables without difficulty by taking failure criterion as an objective function. The design sensitivity with respect to layer angle is analyzed based on the adjoint variable method. It is found that this method provides more precise design sensitivity than F.D.M. In conclusion, the numerical results show that the failure index is minimized successfully and the reasonable optimal stacking sequence design is saught. 


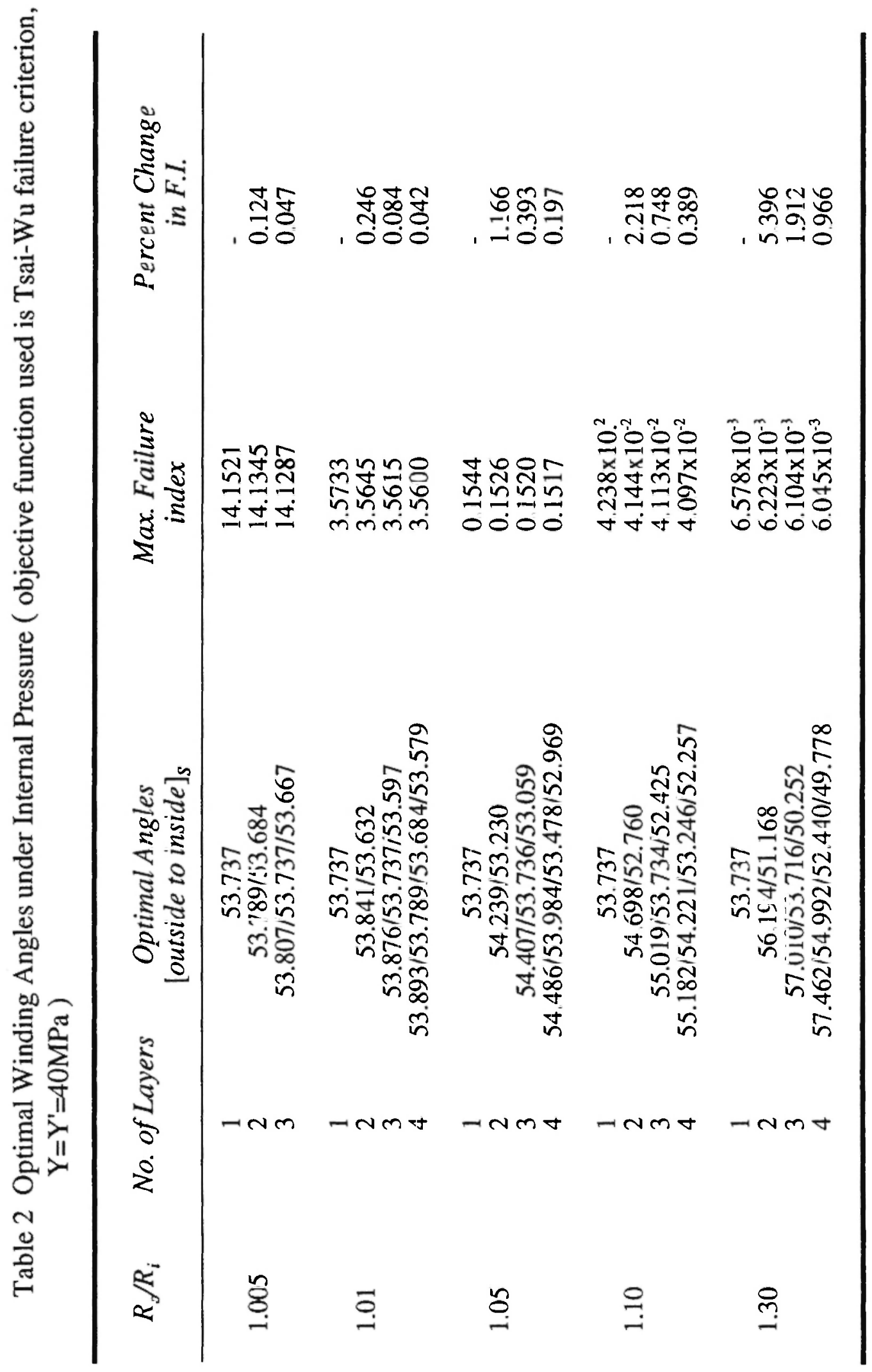




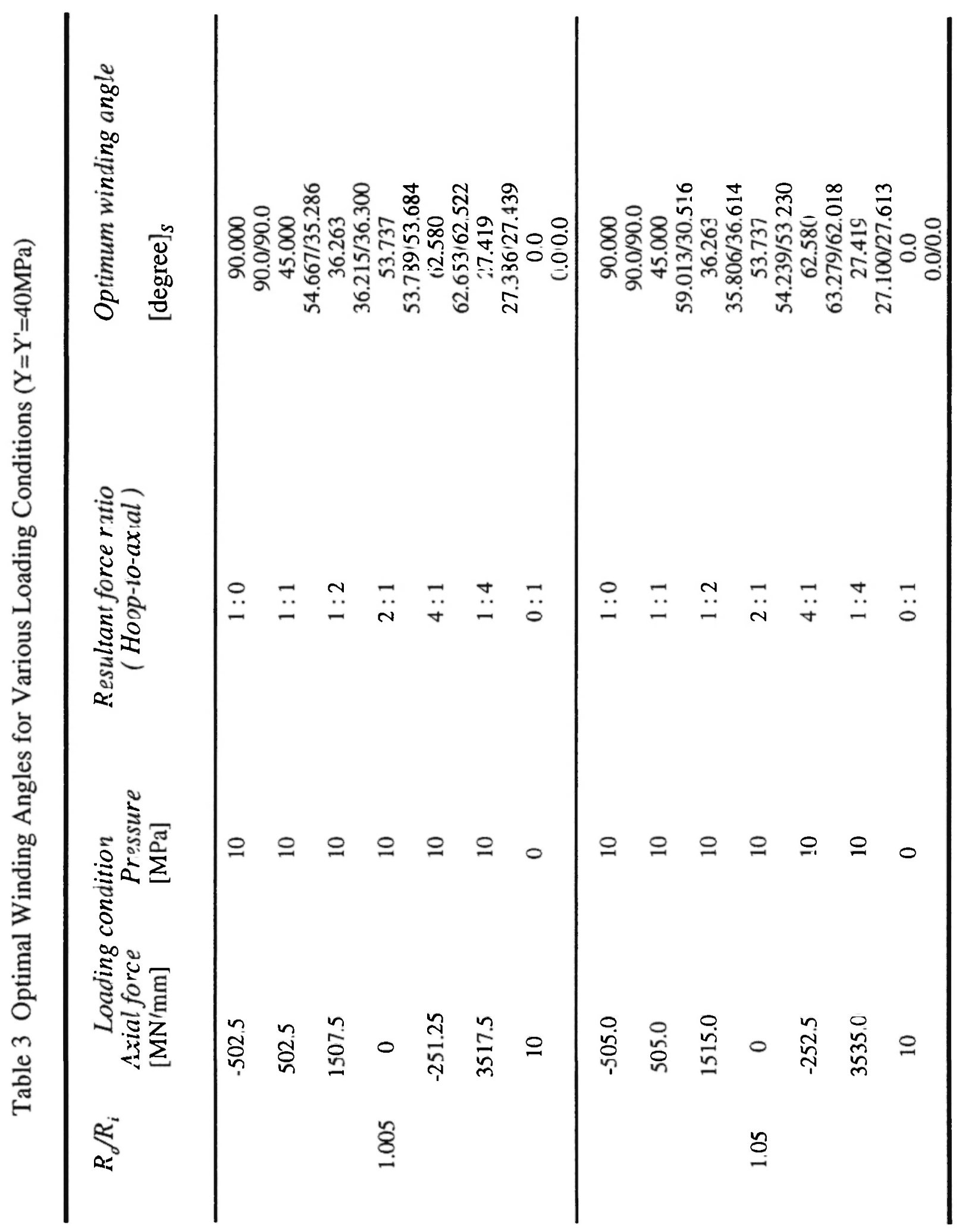




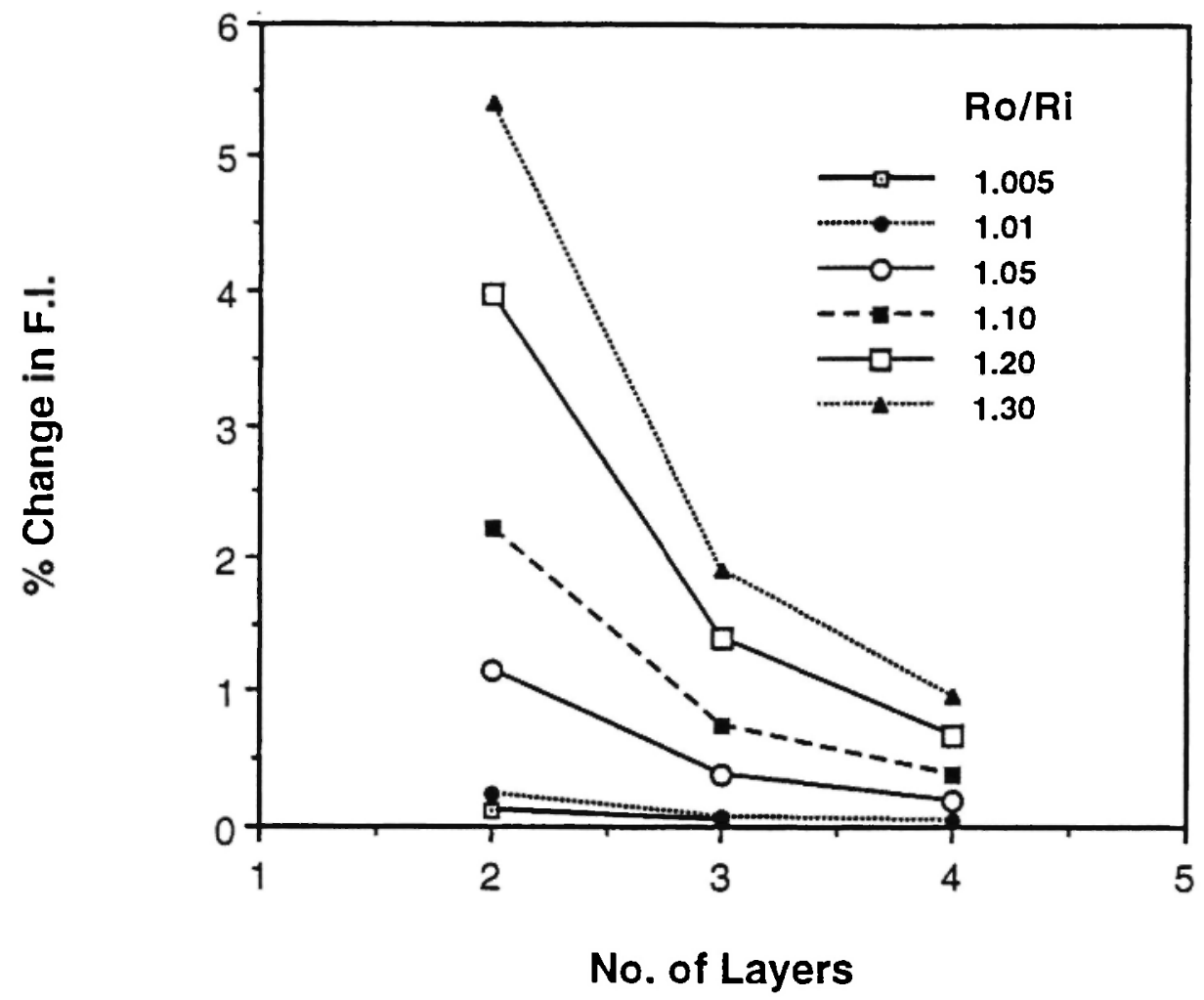

Fig. 6 Percent change in failure index with increasing no. of layers

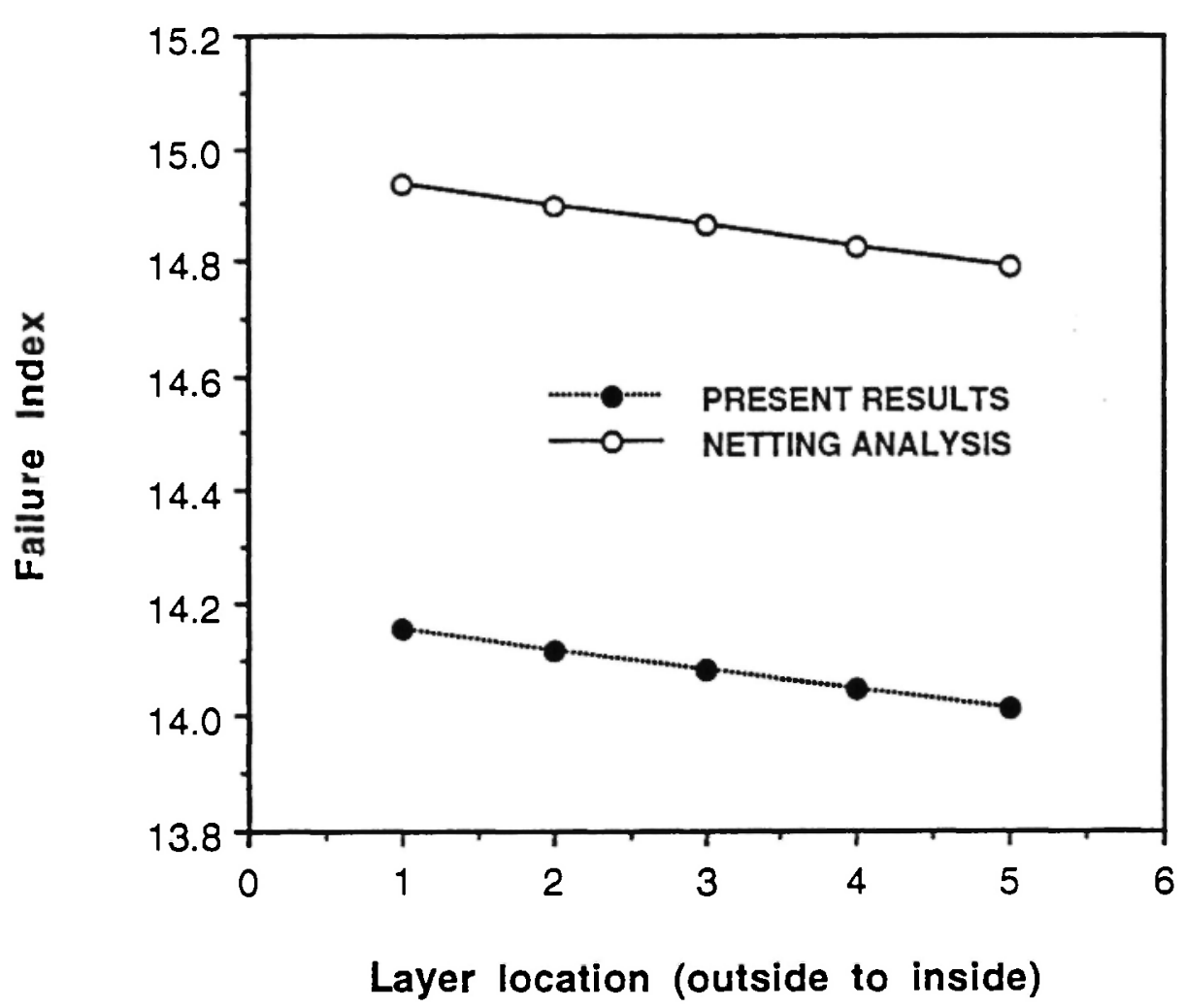

Fig. 7 Comparison of present results with netting analysis $\left(R_{\delta} / R_{1}=1.005, p=10 \mathrm{MPa}\right)$ 


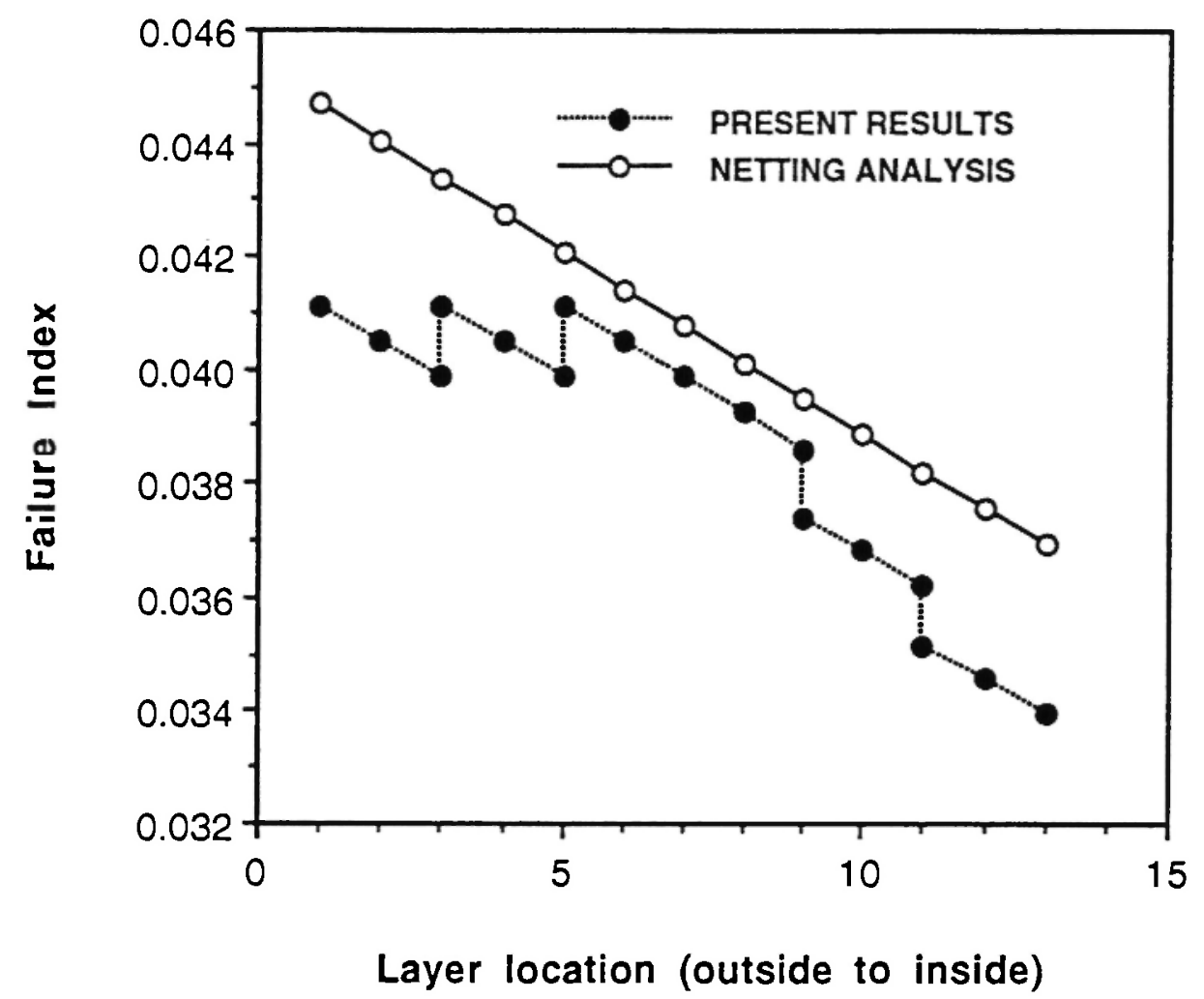

Fig. 8 Comparison of present results with netting analysis $\left(R_{\delta} / R_{i}=1.10, p=10 \mathrm{MPa}\right)$

Table 4 Optimal Angle under Internal Pressure 10MPa with Fixed Inner Layer Angles

\begin{tabular}{ll}
\hline$R_{\delta} / R_{i}$ & $\begin{array}{l}\text { Optimal Angle } \\
{[\theta /-\theta / 90 / 0]_{S}}\end{array}$ \\
\hline 1.005 & 70.787 \\
1.05 & 69.460 \\
1.10 & 68.832 \\
\hline \hline & \\
\hline$R_{\delta} / R_{t}$ & Optimal Angle \\
& {$[\theta /-\theta / 45 /-45]_{S}$} \\
\hline 1.005 & 63.346 \\
1.05 & 63.346 \\
1.10 & 63.346 \\
\hline
\end{tabular}




\section{NOMENCLATURE}

$\begin{array}{llll}\text { A } & \text { extensional stiffness matrix } & \varepsilon^{0} & \text { mid-plane strain } \\ \mathbf{A}^{-1} & \text { inverted extensional stiffness matrix } & Q & \text { transformed stiffness matrix } \\ N & \text { in-plane stress resultants (MN/mm) } & J & \text { Jacobian matrix } \\ p & \text { internal pressure }(\mathrm{MPa}) & b(j+1) & \text { dummy variable } \\ R_{i} & \text { inner radius (mm) } & z & \text { state variable } \\ l^{\prime} & \text { design sensitivity } & \alpha & \text { environmental parameter } \\ h & \text { state equation } & \Psi_{i} & \text { cost function } \\ \theta & \text { layer angle (degree) } & b & \text { design variable }\end{array}$

$\lambda$ adjoint variable

\section{REFERENCES}

1. Schmit, A., Jr., and Farshi, B., Optimum Design of Laminated Fiber Composite Plates, Int. J. for Numerical Methods in Engineering, Vol.11, 1977, pp.623-640

2. Graesser, D. L., Zabinsky, Z. B., Tuttle, M. E., and Kim, G. I., Designing Laminated Composites Using Random Search Techniques, Composite Structures, Vol.18, 1991, pp. $311-325$

3. Tauchert, T. R., and Adibhatla, S., Design of Laminated Plates for Maximum Stiffness, Journal of Composite Materials, Vol.18, 1984, pp.58 - 69

4. Tsai, S. W., Composites Design (4ed.), Think Composites, Dayton, 1988

5. Chao, C. C., Sun, C. T., and Koh, S. L., Strength Optimization for Cylindrical shells of Laminated Composites, Journal of Composite Materials, Vol. 9, 1975, pp.53 - 66

6. Tauchert, T. R., Optimum Design of a Reinforced Cylindrical Pressure Vessel, Journal of Composite Materials, Vol. 15, 1981, pp.390-402

7. Dong, S. B., Pister, K. S., and Taylor, R. L., On the Theory of Laminated Anisotropic Shells and Plates, Journal of the Aerospace Science, Aug., 1962

8. Haug, E. J., and Arora, J.S., Applied Optimal Design, John Wiley, New York, 1979.

9. Haug, E. J., Choi, K. K., and Komkov, V., Design Sensitivity Analysis of Structural systems, Academic Press, Dec., 1984 
10. Tsai, S. W., and Wu, E. M., A General Theory of Strength for Anisotropic Materials, Journal of Composite Materials, Vol. 5, 1971, pp.58 - 80

11. Whitney, J. M., and Halpin, J. C., Analysis of Laminated Anisotropic Tubes Under Combined Loading, Journal of Composite Materials, Vol. 2, 1968, pp.360 - 367 